
3. Kestelyn PH, Stevens AM, Ndayambanje A. HIV and conjunctival malignancies. Lancet 1990;336:512.

4. Bacskulin A, Ehrardt M, Strietzel M, Pau HW, von Schwanewede $H$, Gluthoff $R$. An adjuvant afterloading brachytherapy device for use after orbital exenteration in patients with orbital malignancies. $\mathrm{Br}$ $J$ Cancer 1996 ; 6:484-8

5. Newton R.A review of the aetiology of squamous cell carcinoma of the conjunctiva. Br $J$ Cancer $1996 ; 74: 1511-3$
6. Johnson TE; Tabbara KF, Weatherhead RG, Kersten RC, Rice C, Nasr AM. Secondary squamous cell carcinoma of the orbit. Arch Ophthalmol 1997;115:75-8.

7. Lewallen S, Shroyer KR, Keyser RB, Liomba G. Aggressive conjunctival squamous cell carcinoma in three young Africans. Arch Ophthalmol 1996;114:215-8.

8. Lewallen S, Courtright P. HIV and AIDS and the eye in developing countries. Arch Ophthalmol 1997;115:1291-5.

\title{
Trends in maternal mortality for the Greater Harare Maternity Unit: 1976 to 1997
}

\author{
F MAJOKO, T CHIPATO, V ILIFF
}

\begin{abstract}
Objective: To determine the magnitude, trends and the main causes of maternal death for Harare Maternity Hospital (HMH) and thereby identify potential areas for interventions.

Design: A descriptive retrospective analysis of maternal mortality data from the institution included in publications and recent annual reports.

Setting: Department of Obstetrics and Gynaecology Greater Harare Maternity Unit, Zimbabwe.

Main Outcome Measures: The trends in maternal mortality ratios (MMR) and the relative importance of different causes of death between 1976 and 1997.

Results: There was a decline in MMR between 1976 and the early 1980 s but there has been a steady increase in MMR for Harare residents from 50/100000 in 1988 to 224/100000 in 1997. Sepsis has remained the leading cause of maternal death. There has been a significant increase in indirect deaths due to meningitis, tuberculosis and pneumonia where HIV infection is an underlying factor. Avoidable factors were identified at patient/ community, local health facility and at the tertiary hospital. There has been a decline in the quality of care in recent years.

Conclusion: Maternal mortality for HMH is unacceptably high and could still be rising. HIV infection has contributed to the worsening picture. Interventions to improve access and quality of care at all levels could lead to significant reduction in maternal deaths.
\end{abstract}

Cent Afr J Med 2001;47(8):199-203

\section{Introduction}

The Safe Motherhood Initiative (SMI) launched in Nairobi in 1987 had an objective of reducing maternal mortality ratios (MMR) by $50 \%$ of the 1990 levels by year $2000 .^{1}$ Sub-Saharan Africa has one of the world's highest maternal mortality ratios. ${ }^{2}$ Zimbabwe, like most developing. countries, has incomplete notifications of births and deaths and therefore trends are sometimes derived from health service statistics. The true MMR for Zimbabwe is not known. ${ }^{3}$ The World Health Organisation (WHO) revised 1990 estimates of MMR for Zimbabwe was 570/100 000 whereas the Ministry of Health $(\mathrm{MoH})$ figure for the corresponding year was $82 / 100000.4$

The MMR for Harare Maternity Hospital in 1990 was $80 / 100000$. The differences in rates arise from the method

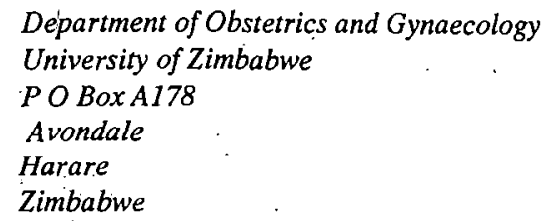

Correspondence to:

Dr F Majoko

e-mail:fmajoko@healthnet.zw 
used to compute the ratio, the coverage and data collection method. The WHO revised 1990 estimates were calculated using national maternal mortality estimates adjusted for under reporting and misclassification. The $\mathrm{MoH}$ calculations are based on death notifications from the provinces and their institutions.

In our setting, there is incomplete notification of deaths especially for women who die at home. ${ }^{3,5}$ In the absence of undisputed reliable national mortality figures, we felt institutional statistics were useful in monitoring trends. Health service statistics, though unrepresentative of the general population, are a reliable source of mortality data for the institutions. Data on maternal deaths have been collected intermittently for HMH but for those years of collection the data are reliable and well analysed. The government health facilities have been well utilised by the majority of women resident in Harare and therefore all maternal deaths within the city would be notified.

We studied the magnitude, trends and causes of maternal deaths in the largest maternity unit in the country to determine factors that could have contributed to the failure of the SMI in not achieving a reduction in maternal mortality in Zimbabwe.

\section{Materials and Methods}

\section{Setting.}

The Greater Harare Maternity Unit (GHMU) is made up of Harare Maternity Hospital (HMH) and the midwife-run municipal clinics. The nurse-midwives in the primary health clinics provide antenatal care and deliver all women with uncomplicated pregnancies. The HMH acts as both a secondary level referral unit for the city clinics and a tertiary referral unit for the district and provincial hospitals in the northern and eastern part of Zimbabwe. Referrals from the provinces account for less than $2 \%$ of all deliveries but for 30 to $60 \%$ of maternal deaths. ${ }^{7-9}$ Women were advised to book for prenatal care and delivery with their local clinic. There are guidelines for referral from the local clinic to the tertiary hospital. Women who meet the indications for referral at any stage of pregnancy are managed at the tertiary hospital. The GHMU has an average of 35000 deliveries per year, 40 to $60 \%$ of which are in $\mathrm{HMH}$. All complicated cases within the Unit are referred to $\mathrm{HMH}$ and therefore most women who die within the city will be included in the hospital statistics.

\section{Methods.}

To study the trends in maternal mortality in the Greater Harare Maternity Unit (GHMU), data from four publications for 1976, 1983, 1987, 1990 and recent annual reports (1991 to 1997) were reviewed. ${ }^{3},{ }^{7,9,10}$ Data for 1977 to 1982 and 1984 to 1986 were not available for analysis. The MMR and main causes of death were compared. Gross MMR for the GHMU and the MMR for Harare residents were calculated. The data in the published reports were extracted from the publications to determine magnitude of maternal mortality and causes for the years reported. A maternal death form has been in use in the unit since 1992 and was last revised in 1994. The data forms for maternal deaths from 1992 onwards were available and were used to extract data for the selected years. In recent years, at least one of the authors has been involved in a review of the clinical notes of maternal deaths. Between 1991 and 1993, the reviews were performed by VI. In 1994 to 1995, all three authors reviewed clinical notes. In 1996 and 1997, FM and TC did the reviews. In determining the cause of death, the reviewing obstetrician made a clinical impression as to the final cause of death and the underlying or contributing factors. The assessor was required to state if the cause of death was clear or not and to classify the death according to category (direct, indirect or coincidental death). In our setting the rate of post mortem to confirm the cause of death was low due to administrative and sociocultural problems. An assessment was also made of avoidable factors at all levels (patient, local health facility and $\mathrm{HMH}$ ). The final cause of death, the underlying factors, category of death and avoidable factors for the years under review were tabulated. In recent years maternal deaths were identified on the obstetric and gynaecological wards by a midwife who visited the wards to inquire about deaths and through death certificates from medical records registry.

\section{Results}

There was a decrease in gross MMR for the GHMU between 1976 and 1983 (Figure I). There was little variation in the MMR for Harare residents between 1976 and 1990. However, there has been a sustained increase in the gross MMR for the Unit and for Harare residents since 1990. The rate of increase was close to $10 \%$ per annum between 1990 and 1994. The MMR appears to have reached a plateau around 350/100 000 for the GHMU and 220/100 000 for Harare residents. Referrals from outside the Unit contributed $39 \%$ to $63 \%$ of maternal deaths.

Figure I: Trends in maternal mortality ratio for Harare residents and for the GHMU 1976 to 1997.

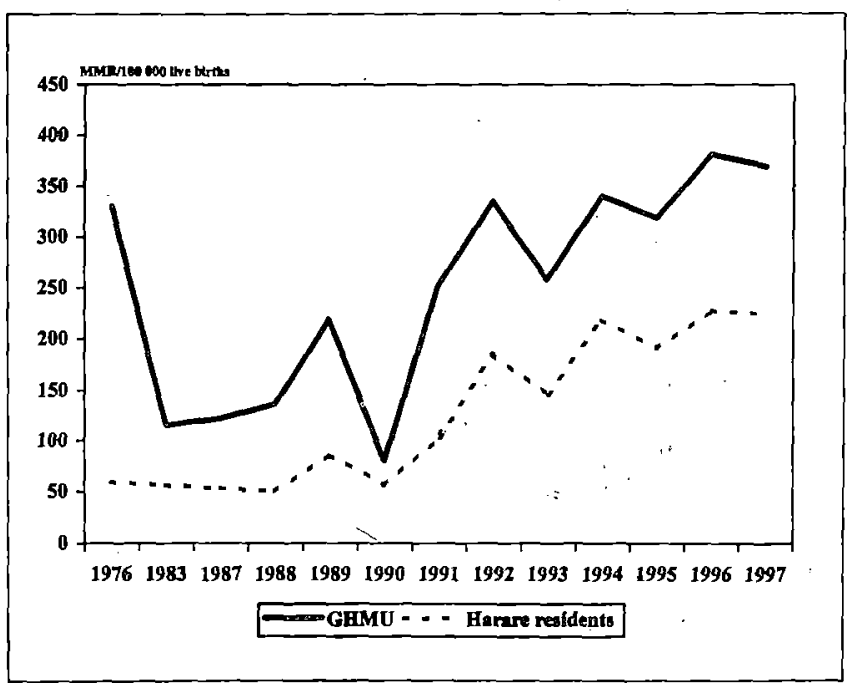


The leading causes of maternal death have been puerperal sepsis, abortion complications, haemorrhage and hypertensive disorders (Figure II). Sepsis has been the leading cause of maternal death accounting for between 22 and $66 \%$ of deaths. In recent years, non-obstetric sepsis has gained more importance with meningitis, pneumonia and tuberculosis responsible for up to $25 \%$ of deaths in some years. Deaths due to puerperal sepsis show a decreasing trend. Abortion complications remain a significant contributor to mortality. There was a decrease in deaths associated with hypertensive disorders of pregnancy between 1987 and 1993 but this trend has not been maintained in the past few years. Deaths due to obstetric haemorrhage decreased significantly between 1987 and 1990 but have since been static.

Figure II: Leading causes of maternal death.



The proportion of indirect deaths has increased from $8.9 \%$ in 1976 to $49.3 \%$ in 1997 (Figure III). The increase in indirect deaths in recent years has been largely due to meningitis, tuberculosis and pneumonia in women with suppressed immunity related to HIV infection. The trend in direct maternal deaths indicates a declining proportion.

HIV infection has been gaining importance as an underlying cause in most indirect deaths (Figure IV). Hypertensive disorders of pregnancy remain a constant underlying cause of maternal death found in 10 to $20 \%$ of cases. Although sepsis has remained the leading cause of

Figure III : Categories of maternal death.

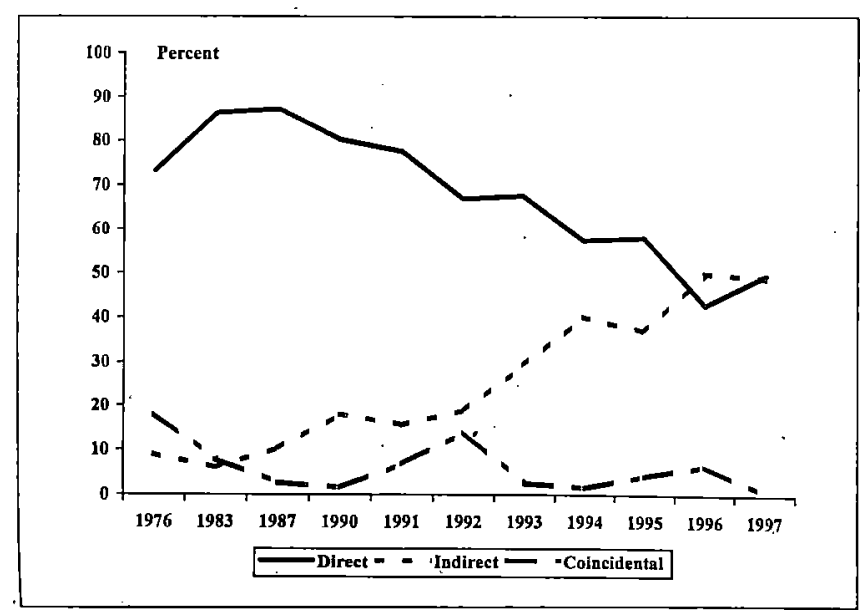

Figure.IV : Major underlying causes of maternal deaths, 1993 to 1997.



death over the two decades, there appears to be a decrease in obstetric sepsis in recent years.

In the majority of maternal deaths there were factors attributable to either the patient or health care system that could have contributed to the bad outcome. These factors included late presentation by the woman, poor clinical management and late transfer by the clinic and delayed diagnosis and treatment in the tertiary hospital. Overall, deficiencies of care were found in more than $60 \%$ of deaths (Figure V). In some patients there were avoidable factors at more than one level.

Figure V: Level of avoidability of maternal deaths for selected years.

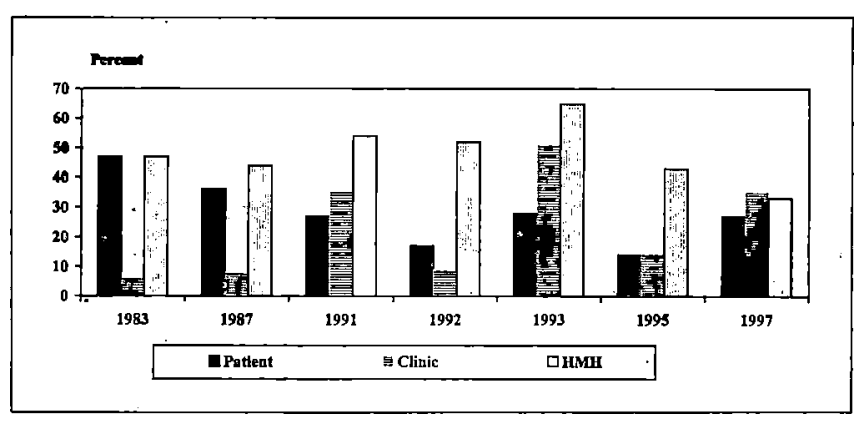

\section{Discussion}

We found that the MMR for Harare residents was stable around 50/100 000 births between 1976 and 1990 but has since increased to around 220/100 000. The gross MMR for the Greater Harare Maternity Unit has increased four fold since 1990 from 90 to $350 / 100000$ live births. The rising trend of maternal mortality in our report is similar to trends from another African setting. ${ }^{11}$ There are, however, reports of declining maternal mortality trends from other low resource areas. ${ }^{12,13}$ The magnitude of maternal mortality problems is obscured by under reporting and misclassification in most developing countries. ${ }^{23,14,15}$

Possible factors associated with maternal mortality increase include changes in either the population, disease patterns or in service delivery. In Zimbabwe, the general 
health of women in the reproductive age group is probably worse than it was in $1980 \mathrm{~s}^{16}$

Puerperal sepsis, haemorrhage, post abortal sepsis and hypertensive disorders were the main causes of death between 1976 and 1990. In recent years, non-obstetric sepsis has accounted for an increasing proportion of deaths. Our finding that infection has been the leading cause of maternal deaths is similar to other reports. ${ }^{12}$ The increase in non-obstetric sepsis indicates both HIV infection and declining quality of care. In our report, deaths due to puerperal sepsis decreased from 34\% in 1976 to $15 \%$ in 1997. A reduction in sepsis-related deaths has also been reported in other settings. ${ }^{12,17}$

There was a decrease in deaths due to hypertensive disorders between 1987 and 1993 but a recent rise in deaths from this cause may be linked to the deteriorating health care delivery system.

Haemorrhage accounted for around 20\% of maternal deaths between 1983 and 1987. Deaths due to haemorrhage were steady around $10 \%$ between 1990 and 1996 .

Mortality from abortion complications decreased between 1990 and 1993 but appears to be increasing since then and this could be a reflection on declining quality of care. There has been deterioration in service delivery partly due to shortages of equipment, drugs and personnel.

Direct maternal deaths decreaséd from almost $90 \%$ in 1983 to $50 \%$ in 1997 but the proportion of indirect deaths in which HIV infection is an underlying factor has been increasing. The shift from direct to indirect deaths has also been observed in other settings. ${ }^{12}$

Post mortems are not frequently performed in Africa for determination of maternal death cause due to socio-cultural and administrative problems. ${ }^{3,11,18}$ In most maternal deaths the cause of death is based on clinical diagnosis. The post mortem rate in maternal deaths for the hospital ranged from $14 \%$ to $28 \% .^{9,10}$ The high post mortem rates of $62.5 \%$ and nearly $100 \%$ reported for maternal deaths occurring in 1972 to 1973 and 1976 respectively were exceptional and 2 may be related to the prevailing political atmosphere in terms of obtaining consent. ${ }^{7,8}$ We do not, however, feel that lack of post mortem diagnosis led to misclassification in the major causes of death.

Cost recovery in the health sector has been associated with an increase in the number of unbooked mothers and a decrease in institutional deliveries. The proportion of unbooked mothers has increased from $6.7 \%$ in 1983 to $12.8 \%$ in $1995 .^{5}$ The mortality in unbooked women has been higher than among women who received antenatal care. $7,8,10,19$

Avoidable factors were identified at all levels i.e. the patient/community, the referring unit and the central unit. Avoidable factors/sub-standard care ranged from $58 \%$ to $88 \% .8,10,19$ High rates of avoidable factors in maternal deaths have been reported from other African settings. ${ }^{11,18}$ The sub-standard factors included: the woman not attending a clinic for antenatal care or delay in presentation; suboptimal care in the referring unit, and inadequate management and delays before surgical intervention in the tertiary hospital.

Potential Areas for Reduction in Maternal Mortality.

The high proportion of sub-standard care in maternal deaths shows that there are possible interventions that could reverse the trend of increasing mortality. Patient education to increase antenatal care attendance and to increase institutional deliveries could reduce mortality, as it has been shown that mortality for unbooked women is much higher than for those who book. ${ }^{19}$ There is need for better equipped staff skilled in managing obstetric complications and improved referral systems. Properly. staffed and equipped central hospitals with the capabilities of dealing with obstetric emergencies are essential in the prevention of maternal deaths. Frequent updates in the skills needed to deal with obstetric complications will improve the quality of care and reduce case fatalities.

Preventable direct obstetric deaths include those from eclampsia, puerperal sepsis, post abortal complications and haemorrhage. Interventions targeting most of these direct causes could result in maternal mortality reduction. Improving the quality of care in women with HIV infection could reduce mortality from non-obstétric sepsis.

\section{Study Limitations.}

This study suffers from the limitations of hospital-based data that are not representative on a national basis. Health service statistics are, however, useful in setting priorities for interventions aimed at reducing maternal mortality. We compared maternal mortality over two decades during which the definition of maternal death changed from a death within 12 months of a pregnancy to 42 days. There was no standardised data form until 1993 and therefore not all variables were studied throughout the period. The assessment of clinical records to determine cause of death has reviewer bias but reviewers tend to become consistent after some time.

Despite these limitations, we believe that maternal mortality for the GHMU remains high.

\section{Conclusion}

Maternal mortality for GHMU is unacceptably high and there is a large potential for reduction through consumer education, improved quality of services and improved access to maternity care. Puerperal sepsis, haemorrhage, post abortal sepsis and hypertensive disorders remain the main causes of maternal death. HIV infection has contributed to an increase in indirect deaths due to nonobstetric sepsis but the magnitude of its effect could be mitigated through improved quality of care.

\section{References}

1. Mahler H. The Safe Motherhood Initiative: call to action. Address for Safe Motherhood Conference. Nairobi, Kenya: World Bank, WHO, UNFPA. 1987.

2. Boerma T. The magnitude of the maternal mortality problem in sub-Saharan Africa. Soc Sci Med 1987;24:551-8. 
3. Fawcus S, Mbizvo M, Lindmark G, Nystrom L and Maternal Mortality Study Group. A community based investigation of causes of maternal mortality in rural and urban Zimbabwe. Cent Afr J Med 1995;41:10513.

4. WHO/UNICEF. Revised 1990 estimates of maternal mortality: a new approach by WHO and UNICEF. Document No. WHO/FRH/MSM/96.11 WHO; Geneva. 1996.

5. National Health Information and Surveillance Unit. Zimbabwe National Health Profile 1998. Ministry of Health and Child Welfare, Zimbabwe. 2000.

6. Oosterhuis JWA. Estimating maternal mortality by sisterhood method in rural Zimbabwe. Trop Doctor 1993;23:67-8.

7. Brown I. Maternal mortality: a survey of maternal deaths occurring during 1976. Cent Afr J Med 1978;24:212-4.

8. MacPherson T. A retrospective study of maternal deaths in the Zimbabwean black. Cent Afr $J$ Med 1981;27:57-60.

9. Crowther C. Maternal deaths at Harare Maternity Hospital during 1983. S Afr Med J 1986;69:180-12.

10. Ashworth MF. Harare Hospital maternal mortality report for 1987 and a comparison with previous reports. Cent Afr J Med 1990;36:209-12.

11. Ujah IAO, Uguru VE, Aisien AO, Sagay AS, Otubu JAM How safe is motherhood in Nigeria?: the trend of maternal mortality in a tertiary health institution. E Afr Med J 1999;76:436-9.
12. Ayhan A, Bilgin F, Tuncer ZS, Tuncer R, Yanik A, Kisnisci HA. Trends in maternal mortality at a university hospital in Turkey. Int $J$ Gynecol Obstet 1994;44:223-8.

13. Kao S, Chen L-M, Shi L, Weinrich MC, Miller CA Maternal mortality in Taiwan: rates and trends. Inter Family Planning Perspective 1997;23:34-5,8.

14. Fawcus S, Moodley J, Bradshaw D, Theron GB, Abdool Karim SS. Measuring maternal mortality in South Africa. S Afr Med J 1996;86:403-6.

15. Orach, CG. Maternal mortality estimated using the Sisterhood method in Gulu district, Uganda. Trop Doctor 2000;30:72-4.

16. Ministry of Health and Child Welfare. Zimbabwe National Health Strategy:1997-2007.

17. Kaupova N, Nukusheva S., Biktasheva H, Goyaux N, Thonneau P. Trends and causes of maternal mortality in Kazakhstan. Int J Gynacol Obstet 1998;63:17581.

18. Moodley D, Payne AJ, Moodley J. Maternal mortality in Kwazulu/Natal: need for an information database system and confidential enquiry into maternal deaths in developing countries. Trop Doctor 1996;26:50-4.

19. Crowther $C$. The prevention of maternal deaths: a continuing challenge. Cent Afr J Med. 1986;32:11-4. 


\section{(c) (1) (9)}

This work is licensed under a

Creative Commons

Attribution - NonCommercial - NoDerivs 3.0 License.

To view a copy of the license please see:

http://creativecommons.ora/licenses/bv-nc-nd/3.0/ 